\title{
COMMENTARX
}

\section{The underuse of digoxin in heart failure, and approaches to appropriate use}

\author{
Ali Ahmed, James B. Young, Mihai Gheorghiade
}

$\infty \quad$ See related article page 644

$\mathrm{M}$ ost of the estimated 400000 Canadians who experience heart failure are 65 years of age or older. Heart failure is the most frequent reason for hospital admission for older adults. Admission for heart failure is a marker of disease progression, a cause of poor quality of life and a burden to the health care system. With the increase of the mean age of the population, the prevalence of heart failure and related hospital admissions are projected to double over the next 2-3 decades.

The largest multicentre randomized clinical trial of digoxin use in heart failure, the Digitalis Investigation Group (DIG) trial, ${ }^{1}$ was conducted in II 6 Canadian and I86 US centres during I99I-I993. It demonstrated that use of digoxin, a digitalis glycoside, was associated with a significant $7.9 \%$ absolute reduction (34.7\% in the placebo group v. $26.8 \%$ for patients receiving digoxin) and $28 \%$ relative reduction (risk ratio [RR] $0.72,95 \%$ confidence interval [CI] $0.66-0.79$ ) in heart failure-related hospital admissions among patients who were also receiving angiotensin-converting enzyme (ACE) inhibitors and diuretics. ${ }^{1}$ Differences in the rate of death from all causes (placebo group $35.1 \%$, digoxin group $34.8 \%$ ) were statistically nonsignificant. ${ }^{1}$

In 1997, the US Food and Drug Administration approved digoxin for use in heart failure. ${ }^{2}$ This approval was based primarily on digoxin's effect on the combined end-points of death due to progressive heart failure or hospital admission due to worsening heart failure, in patients with heart failure (RR 0.69, 95\% $\mathrm{CI} 0.63-0.76$ ) and diastolic heart failure ( $\mathrm{RR} 0.72,95 \% \mathrm{CI} 0.53^{-}$ 0.99) during the first 2 years after group assignment (Table I). ${ }^{2}$

The 2006 Canadian Cardiovascular Society practice guidelines on heart failure recommend the use of digoxin (a class I recommendation, based on level A evidence) to relieve symptoms and reduce hospital admissions among patients with heart failure who are in sinus rhythm and whose symptoms persist despite appropriate medical therapy for heart failure. ${ }^{3}$ Practice guidelines from the American College of CardiologyAmerican Heart Association (ACC/AHA) in 2005 and the Heart Failure Society of America in 2006 make similar recommendations. ${ }^{4,5}$ The ACC/AHA recommendation on the use of digoxin was based on level B evidence, since the data supporting it were derived from a single randomized study, the DIG trial. ${ }^{4}$

Data from large national heart-failure registries and recent trials involving patients with heart failure show a decline in the use of digoxin during recent years. ${ }^{6}$ Possible reasons for this under- use include a focus on mortality rates and lack of attention to hospital admissions as an outcome in cases of heart failure; ${ }^{1}$ perceived concerns about the harmful effects of digoxin in women; ${ }^{7}$ lack of industry promotion and scientific discussions about the role of digoxin in contemporary patients with heart failure who are taking $\beta$-blockers and aldosterone antagonists; and lack of clarity about the role of digoxin in those with diastolic heart failure, who compose up to half of all patients with heart failure.

The primary mechanism of action of digoxin in heart failure is the inhibition of the sodium-potassium adenosine triphosphatase (Na-K-ATPase) enzyme. Suppression of this enzyme in myocardial cells increases heart-muscle contractility. It now appears, however, that the benefit of digoxin in heart failure also results partly from the inhibition of this enzyme in noncardiac tissues. Digoxin reduces sympathetic and renin-angiotensin-aldosterone system activities in heart failure by inhibiting Na-K-ATPase in vagal afferent nerve fibres and the renal tubules, respectively. ${ }^{4}$ The effect of digoxin on neurohormones is believed to be more favourable at low dosages that result in low serum digoxin concentrations. ${ }^{8,9}$ This view is also supported by evidence that, at low serum concentrations, digoxin may reduce rates of death from heart failure, ${ }^{10,11}$ and that the effects of digoxin in systolic and diastolic heart failure are comparable (Table I) ${ }^{1,2,12}$

The role of serum digoxin concentration in digoxin therapy is now well established; major practice guidelines for heart failure recommend that a concentration below $1.0 \mathrm{ng} / \mathrm{mL}$ be achieved for optimum results. ${ }^{3-5}$ However, serum digoxin concentration was considered neither in the main analysis of

Table 1: Effect of digoxin on the combined end-point of hospital admission for worsening heart failure or death from progressive heart failure in patients' first 2 years in the study conducted by the Digitalis Investigation Group

\begin{tabular}{lccc}
\hline Trial & Ejection fraction & Patients & RR $(95 \% \mathrm{Cl})$ \\
\hline Main & $\leq 0.45$ & 6800 & $0.69(0.63-0.76)$ \\
Ancillary & $>0.45$ & 988 & $0.72(0.53-0.99)$ \\
\hline
\end{tabular}

Note: $\mathrm{RR}=$ relative risk, $\mathrm{Cl}=$ confidence interval.

Data are from GlaxoSmithKline's full 2001 prescribing information for Lanoxin (digoxin) tablets, ${ }^{2}$ and Ahmed et al. ${ }^{12}$ 
the DIG trial, which showed a lack of mortality benefit from digoxin, nor the subgroup analysis that suggested potential harm from digoxin in women. ${ }^{1,7}$ In the DIG trial, digoxin concentrations of $0.8-2.5 \mathrm{ng} / \mathrm{mL}$ in serum were considered therapeutic. ${ }^{13}$ Chronic digoxin therapy to maintain serum concentrations above $\mathrm{I} .0 \mathrm{ng} / \mathrm{mL}$ (now considered high and potentially harmful) might have caused the mortality benefit of digoxin in heart failure to be underestimated. ${ }^{1}$ This is also evident from Kaplan-Meier mortality plots, which showed a reduction in mortality rates in the digoxin group during the first year after group assignment. ${ }^{1}$ A recent post-hoc analysis ${ }^{11}$ of the DIG trial demonstrated that digoxin at low serum concentrations may reduce deaths from all causes, as well as hospital admissions due to all causes and due to worsening heart failure (Fig. I).

Because most people with heart failure are elderly and many are women or have chronic kidney disease, digoxin treatment should begin with low doses ( $\leq 0.125 \mathrm{mg} / \mathrm{d}$ ), which is likely to result in low serum concentrations (0.5-0.9 ng/mL). Younger patients with normal renal function who remain symptomatic at this dose may be prescribed $0.25 \mathrm{mg} / \mathrm{d}$ or more. Clinically relevant risk factors for high serum digoxin concentrations ( $\geq$ I ng/mL) include old age, female sex, high levels of serum creatinine, use of non-potassium-sparing diuretics, and digoxin doses of $0.25 \mathrm{mg} / \mathrm{d}$ or more. Patients who have more than one of these risk factors should be administered doses of digoxin no greater than $0.0625 \mathrm{mg} / \mathrm{d} .{ }^{11}$ Except in patients with multiple risk factors for high serum concentrations (e.g., an elderly woman with chronic kidney disease), there is no need for routine monitoring of serum digoxin concentrations. ${ }^{11}$

The evidence for a mortality benefit from low-dose digoxin is derived from post-hoc subgroup analyses. A randomized clinical trial of low-dose digoxin in contemporary patients with heart failure is therefore needed for definitive evidence.

Digoxin can play an important role in the care of contemporary patients with heart failure. It is the drug of choice for the treatment of atrial fibrillation in patients with heart failure. Use of digoxin can effectively reduce occurrences of hospital admission for heart failure, which is considered a marker of disease progression and is associated with poor outcomes. ${ }^{3,6}$ Women with heart failure should not be deprived of digoxin, because

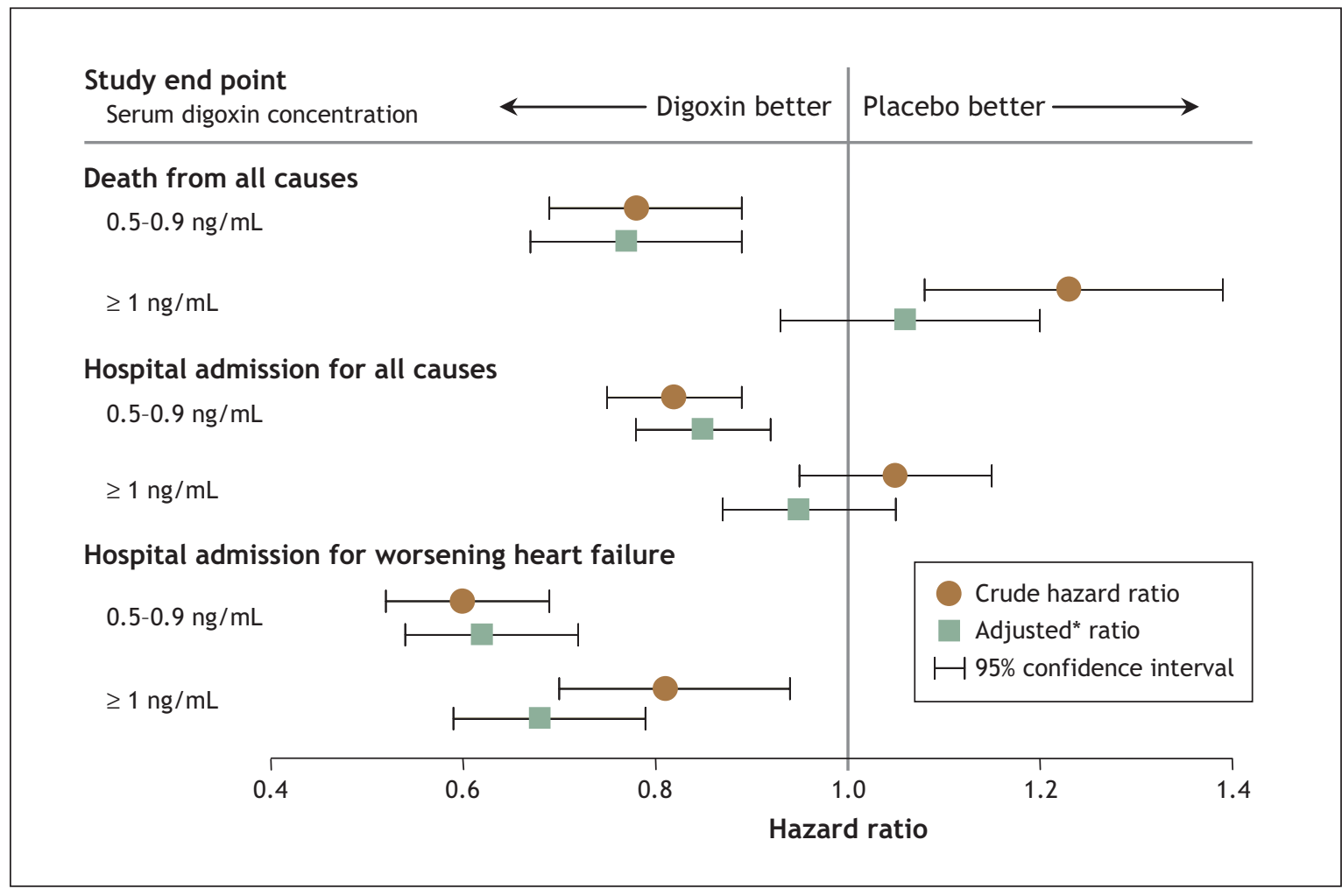

Fig. 1: Effects of digoxin in ambulatory patients with chronic mild-to-moderate systolic and diastolic heart failure in the Digitalis Investigation Group trial. Crude and adjusted hazard ratios for death or hospital admission from any cause and admission due to worsening heart failure are stratified by serum digoxin concentration: $0.5-0.9 \mathrm{ng} / \mathrm{mL}$ versus $\geq 1 \mathrm{ng} / \mathrm{mL}$.

*Adjusted for age, sex, ethnic origin, body mass index, duration and etiology of heart failure, prior myocardial infarction, current angina, hypertension, diabetes, dyspnea at rest and upon exertion, heart rate, systolic and diastolic blood pressure, jugular venous distension, third heart sound, pulmonary rales, edema of the lower extremities, New York Heart Association functional class, pulmonary congestion detected by chest radiography, cardiothoracic ratio above 0.5 , estimated glomerular filtration rate, left-ventricular ejection fraction, drug use (digoxin pre-trial, angiotensinconverting enzyme inhibitors, diuretics, and any combination of hydralazine and nitrates) and an interaction term for serum digoxin concentration $0.5-0.9 \mathrm{ng} / \mathrm{mL}$ and ethnicity (Eur Heart J 2006;27:178-86). 
not only is there no known biological basis for a differential effect of digoxin by sex, but several subsequent analyses have confirmed that when serum digoxin concentration is taken into account, the effect of digoxin in women and men does not differ significantly. Many people with heart failure cannot tolerate ACE inhibitors and $\beta$-blockers, and many in the developing nations cannot afford these drugs. Digoxin may play an important role in the care of these patients. Patients with heart failure in the DIG trial were not receiving $\beta$-blockers or aldosterone antagonists. However, data from spironolactone and carvedilol trials involving patients with heart failure have revealed that digoxin is effective in combination with these drugs. ${ }^{11}$ Finally, digoxin appears to be safe in the treatment of diastolic heart failure. The effect of digoxin in such patients is similar to that of candesartan, which is the only other drug tested in a randomized clinical trial involving patients with diastolic heart failure (Table 2). ${ }^{12,14}$

Over 200 years ago, Dr. William Withering predicted the controversy surrounding digitalis and the expected outcome: "After all, in spite of opinion, prejudice or error, time will fix the real value upon this discovery." ${ }^{15}$ The cumulative evidence from the DIG trial suggests a need for rehabilitation and wider use of digoxin in patients with heart failure. ${ }^{16}$ Digoxin in low doses should be used in patients with heart failure with or without atrial fibrillation if they remain symptomatic despite evidence-based pharmacotherapy with neurohormonal antagonists such as an ACE inhibitor or an angiotensin-receptor blocker, a $\beta$-blocker approved for heart failure (carvedilol or extended-release metoprolol) and an aldosterone antagonist. Digoxin should also be used in patients with heart failure who cannot tolerate or afford these drugs. The role of intravenous digoxin, given its acute hemodynamic effects in patients with acute heart failure syndromes, remains to be determined.

Table 2: Effect of digoxin and candesartan on the combined end-point of hospital admission due to worsening heart failure or mortality due to cardiovascular causes in, respectively, the Digitalis Investigation Group (DIG) Ancillary Trial and the Candesartan in Heart Failure: Assessment of Reduction in Mortality and Morbidity (CHARM)-Preserved Trial

\begin{tabular}{|lcc|}
\hline Characteristic & DIG Ancillary & CHARM-Preserved \\
\hline Drug used & digoxin & candesartan \\
\hline Patients & 988 & 3023 \\
\hline Ejection fraction & & \\
\hline \multicolumn{1}{|c|}{ Inclusion criterion } & $>0.45$ & $>0.40$ \\
\hline Mean & 0.55 & 0.54 \\
\hline Age, mean, yr & 67 & 67 \\
\hline Etiology is ischemic, \% & 56 & 56 \\
\hline Adverse events, no. (\%) & $66^{*}(7)$ & $474 \dagger(16)$ \\
\hline Follow-up, median, mo & 37 & 37 \\
\hline Relative riskł (95\% CI) & $0.88(0.62-1.25)$ & $0.89(0.77-1.03)$ \\
\hline
\end{tabular}

Note: $\mathrm{Cl}=$ confidence interval.

*Suspected or confirmed digoxin toxicity, as identified by study investigators. $\dagger$ An adverse event or laboratory abnormality that caused permanent discontinuation of the study drug.

\#Risk of the combined end-point of hospital admission due to worsening heart failure or death from cardiovascular causes (whichever comes first) in the treatment group, compared with the placebo group.
This article has been peer reviewed.

Ali Ahmed is with the University of Alabama at Birmingham and VA Medical Center, Birmingham, Ala.; James Young is with the Cleveland Clinic Foundation, Cleveland, Ohio; Mihai Gheorghiade is with Northwestern University, Chicago, Ill.

Competing interests: None declared for Ali Ahmed. James Young is a paid consultant for Abbott, Acorn, Amgen, AstraZeneca, Artesion Therapeutics, Biomax Canada, Biosite, Boehringer Ingelheim, Cotherix, GlaxoSmithKline, Guidant, Medtronic, Protemix, Savacor, Scios, Sunshine, Transworld Medical Corporation, Vasogen and World Heart, and has accepted research support from 8 of these and from the US National Institutes of Health. Mihai Gheorghiade is a paid consultant for and has accepted speaker fees from GSK, Medtronic, PDL, Sigma Tau and Otsuka.

Contributors: Ali Ahmed conceived the study hypothesis and design, accessed the data, conducted the analysis and wrote the first draft of the article. All authors interpreted the data, participated in critical revision of the paper and approved the final version to be published.

Acknowledgements: Ali Ahmed is supported by the National Institutes of Health through grants from the National Institute on Aging (I-K23-AGI92II -04) and the National Heart, Lung, and Blood Institute (I-RoI-HLo8556I-OI and P50-HLo77100).

\section{REFERENCES}

I. The Digitalis Investigation Group. The effect of digoxin on mortality and morbidity in patients with heart failure. N Engl J Med I997;336:525-33.

2. Lanoxin (digoxin) tablets [package insert]. Research Triangle Park, NC: GlaxoSmithKline; 200I.

3. Arnold JM, Liu P, Demers C, et al. Canadian Cardiovascular Society consensus conference recommendations on heart failure 2006: diagnosis and management. Can J Cardiol 2006;22:23-45.

4. Hunt SA; American College of Cardiology; Writing Committee to Update the 2001 Guidelines for the Evaluation and Management of Heart Failure, American Heart Association Task Force on Practice Guidelines. ACC/AHA 2005 guideline update for the diagnosis and management of chronic heart failure in the adult: a report of the American College of Cardiology/American Heart Association Task Force on Practice Guidelines (Writing Committee to Update the 200I Guidelines for the Evaluation and Management of Heart Failure) [Published erratum in J Am Coll Cardiol 2005;47:1503-5]. J Am Coll Cardiol 2005;46(6):er-82.

5. Heart Failure Society of America. Executive summary: HFSA 2006 comprehensive heart failure practice guideline [review]. J Card Fail 2006;12:10-38.

6. Gheorghiade M, Zannad F, Sopko G, et al. Acute heart failure syndromes: current state and framework for future research. Circulation 2005;112:3958-68.

7. Rathore SS, Wang Y, Krumholz HM. Sex-based differences in the effect of digoxin for the treatment of heart failure. N Engl J Med 2002;347:I403-II.

8. Slatton ML, Irani WN, Hall SA, et al. Does digoxin provide additional hemodynamic and autonomic benefit at higher doses in patients with mild to moderate heart failure and normal sinus rhythm? J Am Coll Cardiol I997;29:1206-I3.

9. Adams KF Jr, Gheorghiade M, Uretsky BF, et al. Clinical benefits of low serum digoxin concentrations in heart failure. J Am Coll Cardiol 2002;39:946-53.

Io. Rathore SS, Curtis JP, Wang Y, et al. Association of serum digoxin concentration and outcomes in patients with heart failure. JAMA 2003;289:87I-8.

II. Ahmed A, Rich MW, Love TE, et al. Digoxin and reduction in mortality and hospitalization in heart failure: a comprehensive post hoc analysis of the DIG trial. Eur Heart J 2006;27:178-86.

I2. Ahmed A, Rich MW, Fleg JL, et al. Effects of digoxin on morbidity and mortality in diastolic heart failure: the ancillary Digitalis Investigation Group trial. Circulation 2006;114:397-403.

13. The Digitalis Investigation Group. Protocol: trial to evaluate the effect of digitalis on mortality in heart failure. Final protocol. Bethesda, Md.: National Heart, Lung, and Blood Institute; I992.

I4. Yusuf S, Pfeffer MA, Swedberg K, et al. Effects of candesartan in patients with chronic heart failure and preserved left-ventricular ejection fraction: the CHARMPreserved Trial. Lancet 2003;362:777-8I.

I5. Gheorghiade M, Adams KF Jr, Colucci WS. Digoxin in the management of cardiovascular disorders. Circulation 2004;I09:2959-64.

I6. Brophy JM. Rehabilitating digoxin. Eur Heart J 2006;27:I27-9.

Correspondence to: Dr. Ali Ahmed, University of Alabama at Birmingham, 1530 3rd Ave. S, CH-rg, Ste. 219, Birmingham AL 35294-204I, USA; fax I 205 975-7099; aahmed@uab.edu 